\title{
Lumostatic operation controlled by the optimum light intensity per dry weight for the effective production of Chlorella zofingiensis in the high cell density continuous culture
}

\begin{abstract}
To maximize the production rate of Chlorella zofingiensis, a lumostatic continuous culture was operated under light intensities of $250-1510 \mu \mathrm{E} \mathrm{m}^{-2} \mathrm{~s}^{-1}$. The cell density and volumetric biomass production rate were increased without photo inhibition and reached $13.5 \mathrm{~g}$-dry weight $(\mathrm{dw}) \mathrm{L}^{-1}$ on day 21.5 and $2.41 \mathrm{~g}-\mathrm{dw} \mathrm{L}^{-1}$ day $^{-1}$ on day 10.5 , respectively. These maximum values were higher than any previous photoautotrophic culture study with $\mathrm{C}$. zofingiensis. The specific growth rate was maintained at a high level $>0.5 \mathrm{day}^{-1}$ until the

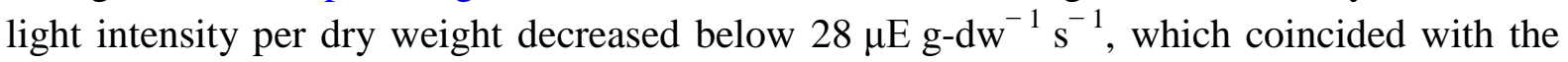
value estimated in our previous study, verifying the reliability of this estimated value. There was a strong relationship between the photosynthetic efficiency and light intensity per dry weight for C. zofingiensis. This relationship may be useful for evaluating species-specific productivity to select productive species.
\end{abstract}

Keyword: Lumostatic operation; Chlorella zofingiensis; High-cell-density culture; Light intensity per dry weight; Continuous culture 